LOS CANALES DE REPRESENTACION SENSORIAL Y LAS ESTRATEGIAS DE APRENDIZAJES UNIVERSITARIOS EN EL INGRESO A LA CARRERA DE MEDICINA VETERINARIA.

FASCENDINI, Paola Y. ${ }^{1}$

${ }^{1}$ Servicio Orientación Educativa.

Facultad de Ciencias Veterinarias. Universidad Nacional del Litoral. Esperanza, Santa Fe. Argentina. 3080. (54) 03496-420639.pfascendini@fcv.unl.edu.ar 


\title{
LOS CANALES DE REPRESENTACION SENSORIAL Y LAS ESTRATEGIAS DE APRENDIZAJES UNIVERSITARIOS EN EL INGRESO A LA CARRERA DE MEDICINA VETERINARIA.
}

\author{
FASCENDINI, Paola Y. ${ }^{1}$ \\ ${ }^{1}$ Servicio Orientación Educativa.
}

Facultad de Ciencias Veterinarias. Universidad Nacional del Litoral. Esperanza, Santa Fe. Argentina. 3080. (54) 03496-420639.pfascendini@fcv.unl.edu.ar

\section{Resumen:}

La investigación apunta a caracterizar los canales de representación sensorial y las estrategias de aprendizajes utilizadas por los estudiantes ingresantes a la carrera de Medicina Veterinaria de la Facultad de Ciencias Veterinarias, dependiente de la Universidad Nacional del Litoral. Particularmente se identificó aquellos canales y estrategias de aprendizajes autodeclaradas por los ingresantes, buscando explorar las razones por las cuales eligen determinadas estrategias para estudiar. La metodología de abordaje fue un enfoque mixto. Desde las variables cuantitativas se administró una encuesta sobre una muestra selectiva de juicio o intencional. Se complementa dicha información con entrevistas realizadas a ingresantes, junto a la administración de cuestionarios y entrevistas a docentes. Se suma a la investigación los aportes brindados por la técnica de talleres formativos realizados en el ingreso universitario. Atendiendo a las conclusiones, se registra en los ingresantes el uso de diversos canales de aprendizaje, siendo el canal visual el más utilizado. La estrategia de aprendizaje más elegida es el resumen sobre la base de la técnica del subrayado. Los motivos por los cuales los alumnos eligen estas estrategias se vinculan con la mecanización utilizada en formaciones anteriores. Si bien los docentes reconocen su valor no se instrumentan en las planificaciones didácticas. 
Palabras claves: canales de representación sensorial, estrategias de aprendizajes, universidad, ingreso.

\section{Summary}

This study aims at characterizing the channels of sensory representation and learning strategies used by freshmen students that enroll in the degree course of Veterinary Medicine at the Faculty of Veterinary Sciences (Universidad Nacional del Litoral). In order to explore the reasons why certain study strategies are specially chosen, particular emphasis was placed on the identification of the learning channels and strategies that freshmen use. The methodology chosen consisted of a mixed approach. A survey was used and sent to a purposive sample using the quantitative variables. This information was complemented with interviews to freshman students, along with questionnaires and interviews to teachers. The information gathered from the training workshops carried out during university admission was also considered. When conclusions are addressed, it is clear that freshmen use diverse learning channels, but the most widely used channel is the visual one. As regards learning strategies, most of them choose to summarize contents by underlining them. The reasons why students choose these strategies are linked to the mechanization of previous trainings. Although teachers recognize the value of these strategies, they are not implemented in their didactic planning.

Keywords: channels of sensory representation, learning strategies, university, freshmen enrollment.

\section{Introducción}

El creciente número de estudiantes que presentan bajos rendimientos académicos o no finalizan sus estudios universitarios, interpela a los educadores y los desafía a ampliar y enriquecer la mirada sobre esta problemática. "Algunos docentes vierten quejas porque la información que imparten no llega a todos de manera efectiva y se preguntan por qué algunos alumnos logran comprender y otros no" (Fernández Coto, 2012. p.94), generalmente aludiendo a su falta de atención. Una posibilidad es que en dicho momento del encuentro áulico, estos estudiantes no estaban sincronizados con los canales de representación sensorial que utilizaba el docente, generando como corolario la falta de comprensión del tema explicitado. 
Cada vez que se explica algo o que se le brinda a los alumnos un ejercicio, se utiliza un sistema de representación y no otro. "Cada ejercicio, cada actividad, cada experimento, según como esté diseñado, presentará la información de una determinada manera y le demandará a los estudiantes que utilicen unos sistemas de representación concretos" (Neira Silva, 2016,p.1).

Estos sistemas y/o canales de aprendizaje se presentan bajo formatos visuales, auditivos, olfativos, gustativos y kinestésicos. Mirando la diversidad estudiantil, se observa que el alumno visual requiere del uso de imágenes, gráficos, esquemas, mapas conceptuales, organizadores gráficos, titulaciones, colores para poder aprender. El auditivo se vincula con la palabra audibilizada, conversaciones, sonidos almacenados, uso del diálogo interno/autodiálogo, el gustativo con los recuerdos del sabor, el olfativo con olores, perfumes y el kinestésico que referencia todo aquello relacionado con lo motriz, el movimiento, el hacer, las sensaciones táctiles.

Cada persona tiene un estilo preferido de reunir, almacenar, recuperar y comunicar la información .La importancia de estos sistemas y canales de representación radica en que al conocer el sistema dominante de una persona, se está en mejores condiciones de adaptar los mensajes a las necesidades de la misma, empleando palabras y gestos que resultan aún más efectivos para el proceso de comprensión.

Es importante destacar que el hecho de desarrollar un canal más que otro, no significa la anulación de los demás, sino que es una preferencia de canal dominante (O’Brien, 1989). El interés por los canales de aprendizaje ha vuelto a tomar fuerza para encontrar una relación entre la manera en la cual los estudiantes perciben la información y el modo en cómo se apropian de ella, observando dificultades y posibilidades (Min y Jie 2005), (Fleming y Baume 2006), (Valdivia 2011), para poder cubrir así los diversos sistemas que porta cada uno, asegurando la práctica multisensorial en respeto por la propia manera de aprender, ya que allí se encuentra la clave del éxito. (Fernández Coto, 2012).

El uso de estrategias de aprendizaje acordes a la modalidad del mismo influye en la persistencia de la tarea, en la comprensión y en una efectiva autoregulación.

No sólo los alumnos tienen sus preferencias y su propio canal más potenciado, sino que todos los profesores tienen inclinaciones electivas vinculadas con ellos, las que le permiten sentirse cómodos en ese estilo propio de dar clase, reflejando formas, procesos y herramientas acorde con dicha modalidad. "La mayoría de nosotros tendemos a utilizar más de un sistema de representación que otro, cuando damos clase" (Neira Silva, 2016,p.2), por resultar ser el que se encuentra en sintonía con los modos de aprender experimentados a lo largo de toda la formación educativa. Además los propios docentes, mediante la aplicación de estrategias y canales de aprendizaje, 
dentro de la praxis pedagógica, logran reconocer los propios procesos cognitivos, ayudando a los estudiantes a alentar y cultivar una disposición favorable para la reflexión, condición intrínseca de todo saber (Fourés,2011), generando modificaciones en la forma de enseñar, para producir cambios en la manera de aprender.

El concepto de estrategias de aprendizaje y canales de representación sensorial, resultan altamente atractivo, ya que aportan basamentos teóricos vinculados a diversas aplicaciones prácticas, permitiendo detectar obstáculos, preferencias al momento de aprender para construir así una propia identidad cognitiva, en pos de beneficiar no sólo la transferencia de saberes a otros contextos, sino fundamentalmente consolidar un aprendizaje duradero y profundo. Cuanto mayor conocimiento posea el docente acerca de los procesos llevados a cabo por el estudiante, mayores serán las posibilidades de acercar el saber a los propios códigos, ajustando el comportamiento (lo que piensa y hace) a las exigencias de la tarea y actividad como también a las circunstancias contextuales.

Es en este contexto que surge el interés por identificar, los canales de representación sensorial y estrategias de aprendizajes idóneos, a fin de poder instrumentar métodos de enseñanza eficaces por parte del docente, tales que se logre un rendimiento académico significativo en los contenidos de todas las áreas, despertando el interés del estudiante no sólo en las áreas de formación general sino también en las de formación de especialidad.

Emergen entonces los siguientes interrogantes: ¿Cómo instaurar una experiencia educativa que permita al alumno y al docente conocer?, ¿Cómo se aprendió lo que se aprendió?, ¿Cómo ocurre la construcción del propio método de estudio?,¿Qué procesos posibilitan los canales y estrategias para el logro de los aprendizajes?, ¿De qué modo aprenden lo que aprenden?, ¿Qué canales de aprendizaje y estrategias emplean los ingresantes al realizan un estudio universitario?, ¿Cuáles son las razones por las que eligen determinadas estrategias para estudiar?.

Por estas razones, se justifica la presente investigación debido a la impronta trascendental que adquiere la temática, al ramificarse en las prácticas educativas y promover la generación de cambios a nivel conceptual, actitudinal y estratégico en el diseño de las metodologías docentes, además de impactar notablemente en la formación de paradigmas educativos de autogestión.

\section{Metodología}

Teniendo en cuenta las características del objeto de investigación, referido a los canales de representación sensorial y estrategias de aprendizajes en el nivel 
universitario, resultó indispensable para su intervención, aplicar una metodología mixta Creswell (2009) que contempla procedimientos concurrentes, es decir "aquellos que se utilizan en forma simultánea con abordajes cuantitativos y cualitativos, consiguiendo así una mejor comprensión del objeto de estudio" (Rodríguez Gómez, 2001,p.14). Este encuadre metodológico permitió indagar los canales existentes y estrategias de apropiación del saber con las que ingresan los estudiantes y de las que se sirven para aprender, triangulando información vinculante tanto a las dimensiones cualitativas como cuantitativas. En cuanto a las clasificaciones sobre diseños de método mixto, planteados en la bibliografía, la investigación realizada, respondió a los siguientes criterios: Diseño de método mixto, de estrategia concurrente de triangulación CUAL $\rightarrow$ Cuanti-(Johnson y Onwuegbuzie, 2004). En cuanto al tipo de estrategia seguida, la investigación respondió a una secuencia descriptiva que aborda dimensiones CUALITATIVA-cuantitativa, con integración de datos en la interpretación y cuyo énfasis fue explicar e interpretar relaciones (Creswell, 2003). El diseño mixto (DIMIX) utilizado contempló dos fases, las que, por cronología en la aplicación, se han definido como fase I y fase II. La fase I respondió a un enfoque cualitativo, el cual, temporalmente, se desarrolló primero. La segunda, dentro de un enfoque cuantitativo, el que, para su diseño y desarrollo, se nutrió de la fase I. La fase I conllevó la utilización del método descriptivo Hernández (2003), citando a Danke, afirman que los estudios descriptivos “(...) buscan especificar las propiedades, las características y los perfiles importantes de personas, grupos comunidades o cualquier otro fenómeno que es sometido a un análisis" (p. 117). Con la premisa de que el estudiantado universitario tiene mucho que aportar en la comprensión de las estrategias y canales de aprendizajes, sabiendo que las experiencias de formación universitaria experimentadas, les faculta para ofrecer opiniones válidas, acerca de las características que asumen las estrategias de aprendizajes utilizadas y la preferencia por los canales para aprender. Por ello en dicha fase se administraron entrevistas personalizadas que permitieron recopilar rasgos representativos de los ingresantes a la hora de estudiar, elaborando un mapeo del objeto de estudio en función de las características y motivos por los cuales utilizan determinadas estrategias para aprender y prefieren ciertos canales de aprendizaje. La selección de los estudiantes estuvo situada sobre la técnica de la bola de nieve que permitió el acceso al modo en que ellos ven, clasifican y experimentan el mundo (Taylor y Bogdan, 1990,p.114).Se registraron los datos bajo la técnica de frases más significativas (Sirvent, María Teresa, 1998) de los entrevistados, transcriptos textualmente y coronados en categorías analíticas. Además en dicha fase se introdujo la mirada de los docentes para reconocer qué tipo de conocimiento poseen acerca de los canales de 
representación sensorial y la utilización que hacen de los mismos. Se previó la aplicación de un cuestionario focalizado en relación con los modos que los profesores tienen al enseñar, para poder reconocer la existencia y-o presencia de los canales de representación sensorial y las estrategias de aprendizajes en las clases que dictan. El mismo constó de seis preguntas, las que se subdividieron en varios sub-ítems por cada interrogante, (a razón de tres), las que combinan preguntas abiertas y cerradas sobre cuestiones centrales en relación con el conocimiento, aplicación, programación de clases y encuentros con los estudiantes sobre la base de los canales de aprendizaje y estrategias. Los primeros interrogantes apuntaron a indagar el conocimiento que tienen los docentes respecto de la temática y sus implicancias. Luego se incorporaron consultas en relación a la inclusión de acciones didácticas que propician los procesos y canales cognitivos, como así también la identificación de actividades que contemplen este formato. Otras consultas refirieron a las estrategias que ellos como docentes verifican como las más eficaces para favorecer el aprendizaje de las disciplinas que dictan. Culminó la misma con cuestiones vinculadas al clima de clase y procesos autoreflexivos instaurados en la práctica diaria. La presentación del cuestionario fue diseñado bajo el formato de documento google drive. También se incluye en el análisis la técnica de talleres formativos que instauren el debate y análisis de los procesos cognitivos que administran los estudiantes a la hora de estudiar, como también incorporar otras variables vinculantes a la historia en los aprendizajes, herramientas utilizadas, formación en la secundaria, aprendizajes previos, canales de aprendizaje de preferencia, valor de las herramientas de estudio, aportando desde las experiencias previas, un análisis de la modalidad realizada para evaluar su pertinencia en el uso. El análisis propuesto para realizar la triangulación constó de dos talleres realizados con comisiones de 70 ingresantes. Para la dimensión cuantitativa se procedió a la recolección de información, mediante la aplicación de una encuesta a ingresantes, de la cohorte 2016, (mediante un link y en formato google drive que permite sintetizar la información relevada). Se redacta un formulario como documento google drive, en formato de encuesta, en el que se indaga acerca de modos, canales de aprendizaje y uso de estrategias para aprender con un total de 30 preguntas, las que se subdividen en categorías vinculadas a:

1) Cuando asisto a clases: refiere a todos los aspectos que se involucran en el proceso de selectividad atencional que una clase requiere y a los procesos cognitivos que en ella ocurren.

2) Antes de ponerme a estudiar: usos organizativos, distribución temporal.

3) Cuando me pongo a estudiar: abarca las estrategias de aprendizajes y canales de 
representación sensorial utilizados al momento de aprender.

4) Cuando realizo trabajos solicitados por profesores: usos de escritura, comprensión de consignas, interpretación.

5) Los trabajos prácticos y mi especial forma de ser: preferencia de usos escritos u orales, entrega a tiempo, contemplación de valoraciones ajenas.

\section{Resultados}

Atendiendo a las conclusiones, se registra en los ingresantes el uso e instrumentación de diversos canales de aprendizaje, siendo que el canal visual es utilizado siempre por el $55,4 \%$ de los estudiantes. Aquellos que eligen el canal auditivo lo utilizan parcialmente en un $43,2 \%$, resultando de preferencia el canal visual. La estrategia de aprendizaje más elegida es el resumen en un $65,5 \%$ sobre la base de la técnica del subrayado. Dicha estrategia aparece como la más utilizada, el $71,6 \%$, siendo que los mapas conceptuales, esquemas y gráficos sólo son instrumentados parcialmente en un $54,4 \%$. Los motivos por los cuales los alumnos eligen estas estrategias se vinculan con la mecanización utilizada en formaciones anteriores, existiendo poca relación entre los canales más desarrollados de sí mismos y las estrategias compatibles a dicho canal. Si bien, los docentes y los estudiantes reconocen la importancia del valor que tiene aplicar estos canales de aprendizaje y su vinculación con las estrategias relacionadas, no se aplican en los procesos de enseñanza y aprendizaje, ya que los mismos se sitúan sobre paradigmas enciclopedistas que obstaculizan la puesta en marcha de estas intervenciones didácticas, centrando la mirada en la memorización de los saberes, más que en la reflexión de procesos de aprendizaje autoregulados. Existe un uso parcial tanto de los mapas conceptuales, gráficos, esquemas, como de la lectura y relectura del texto como estrategia de aprendizaje. La utilización de las explicaciones orales ha sido otra estrategia de aprendizaje muy usada por los ingresantes, como así también el uso del repaso de la información como otro modo de corroborar lo aprendido. Otra estrategia de aprendizaje administrada se encuentra en la inclusión de las sugerencias vertidas por docentes ante la realización de trabajos prácticos, siendo la consulta al docente un camino de menor uso. Si contemplamos el Eje III, que se vincula específicamente con los objetivos de la investigación: estrategias y canales de aprendizaje, podemos apreciar las siguientes consideraciones a partir de la presentación gráfica. El gráfico muestra en relación con la pregunta N 11 que la lectura y relectura de los textos es realizada parcialmente entre el 40 y $55 \%$, evidenciando modos mecánicos de intervención ante el texto de estudio. Al contemplar la pregunta N 12 y 13 observamos que entre el $65 \%$ y $80 \%$ de los estudiantes utiliza la 
técnica del subrayado como un modo de acortar información y elaboración de la técnica del resumen para poder aprenderla (entre $55 \%$ y $70 \%$ responde totalmente de acuerdo). La pregunta N 14 referente al uso e implementación de mapas conceptuales, gráficos, esquemas como estrategias que favorecen la comprensión, entre el $40 \%$ y $55 \%$ de los estudiantes se encuentra parcialmente de acuerdo con dicho uso. En relación con el uso del canal visual para poder aprender, entre el $45 \%$ y $60 \%$ aprende mediante imágenes, siendo que entre el $35 \%$ y el $53 \%$ se encuentra parcialmente de acuerdo con el uso de la memoria auditiva para poder aprender los textos de estudio. Entre el $55 \%$ y $70 \%$ de los estudiantes realizan repaso de la información como un modo de asegurarse de lo comprendido y-o aprendido, resultando un tanto inconsistente el uso que se hace de las estrategias de aprendizajes en relación con los canales o modalidades para poder aprender. Entre el $45 \%$ y $60 \%$ refieren prácticas de revisión de lo aprendido, si consideramos que en una gran mayoría eligen transcribir las ideas en formato de resumen como estrategia elegida por excelencia. Si bien la estrategia reúne características visuales, sabiendo que la mayoría opta por esta modalidad de aprendizaje, dicha elección recae en un uso técnico más que estratégico, por resultar ser la más conocida y utilizada en contextos anteriores de formación, sin incluir otros recursos visuales que contribuirían a un estudio más reflexivo como es la administración de mapas conceptuales, esquemas de relación conceptual, entre otros.

Gráfico de intervalo de confianza del Eje III 


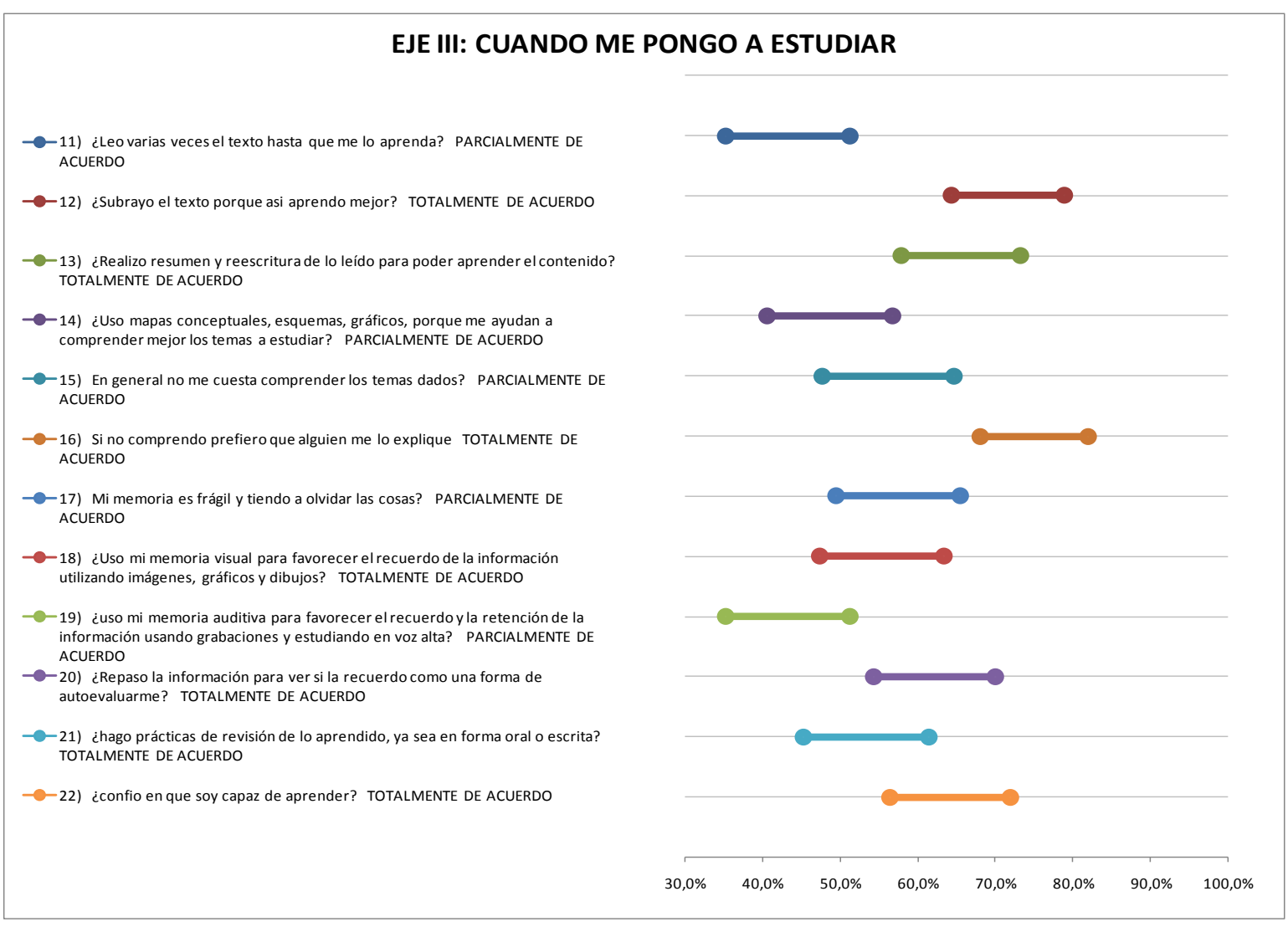

\section{Discusión}

Queda como punto de discusión si en la realidad del aula, el docente puede atender a la diversidad de los canales de aprendizaje que se presentan en la clase y si es posible el diseño de estrategias de enseñanza que involucren todos estos estilos, lo cual revierte cierto grado de complejidad y de conocimientos pedagógicos y didácticos sólidos que le permitan al docente no perder de vista la responsabilidad que tiene en los procesos de enseñanza de la disciplina. Una de las desventajas de muchos de los docentes universitarios para afrontar este reto es el no tener una formación pedagógica adecuada, pues su competencia mayor está en el campo disciplinar, más que en el campo didáctico. Si consideramos que algunos estudiantes, parcialmente reflexionan sobre lo que han aprendido, sumado a la escasez de estrategias de aprendizajes que administran, con un escaso nivel de organización personal, existe una probabilidad muy alta que estos aprendizajes se construyan sobre la base de la repetición y mecanización por la carencia vincular expresada en el uso escaso de estrategias que permitan favorecer las relaciones y redes de conceptos. Por tanto, una estrategia de enseñanza deberá trabajar en función de los canales de percepción para que el estudiante diversifique la manera cómo aprende -sus canales de aprendizaje-, (Mora 2015) que en últimas, es susceptible de mejorar si consigue explorar distintas formas de aprender. En este sentido uno de los principales desafíos que las 
universidades deben enfrentar, es el de analizar cómo el estudiante con la diversidad actual, mejora sus aprendizajes auto gestionándolos tanto dentro como fuera del aula. En relación con ello es importante agregar que el docente es un factor decisivo para alcanzar estos objetivos (Unesco, 2014), aunque no resulta claro aún cómo se pueden brindar las herramientas a los profesores para que lo logren. Es evidente que el mejor camino y apuesta es aportar al desarrollo profesional compartiendo estrategias que se pueden repicar y mejorar en el aprendizaje de los estudiantes. Se enfatiza la necesidad de considerar aportes de (Dunn y Dunn, 1985), quienes advierten que las personas perciben el mundo desde tres canales de percepción, siendo potencial (Quiñonez, 2004) el desarrollar estrategias de aprendizaje y enseñanza de una forma coincidente con el modo en que el estudiante percibe el mundo y asegurando la gran diversidad de aprendizajes.

\section{Conclusiones}

Los canales de aprendizaje que han sido utilizados por los estudiantes, muestran inclinación hacia usos multisensoriales en los que se encuentros los visuales, auditivos y kinestésicos (existiendo cierta preferencia por el canal visual) de acuerdo a la modalidad más conveniente, en cuanto al tipo de memoria que se trate y los canales de aprendizaje más utilizados en etapas previas de su formación. Los canales de representación sensorial resultan conocidos, pero poco potenciados, por rutinas instaladas y faltas de movilidad en el caminar estratégico de aprendizaje. Además las estrategias más utilizadas a la hora de aprender el contenido se vinculan con el uso de resúmenes y subrayados como herramientas y estrategias casi exclusivas e indispensables en el proceso de apropiación, cayendo en la repetición y mecanización de los saberes, con escasez de implicancias relacionales y conectivas. El uso de conexiones y redes como así también la comparación es una herramienta poco utilizada en los aprendizajes universitarios de los estudiantes ingresantes a la carrera de Medicina Veterinaria. La elección de los motivos o razones por las que se inclinan a éstas estrategias de estudio se vinculan con la repetición, mecanización y se encuentran asentadas sobre la base de aquello que es conocido, por ello al ser trasladadas a otro contexto formativo, se produce una escasez de autocrítica y análisis, como así también una falta de puesta a prueba de otras herramientas para el estudio, por contemplar el acostumbramiento como el eje de la elección y la proyección continua de dicha perspectiva, aunque cambien los formatos, los textos o el contexto. Si triangulamos la información podemos vislumbrar estudiantes que saben qué canales de aprendizajes usan para aprender mejor, pero administran estrategias de estudio que no favorecen ni redes ni relaciones entre conceptos, sumada a la 
escasez reflexiva, con intencionalidades docentes propiciadoras de espacios formativos constructivos, pero que en la acción concreta no se corresponden con las estrategias propias de dichas teorías del aprendizaje, por lo que deja perplejo al alumno a recurrir a estrategias de estudio mecanizadas por encontrarse en un terreno memorístico y enciclopedista, atravesado por discursos innovadores pero nada propiciantes de aquello que marketinean en los procesos de enseñanza y aprendizaje. El estudiante que debería experimentar estas vivencias sólo reproduce aquello que le permite responder a las exigencias tradicionalistas concretas, que no resultan ser más que una prolongación de la experiencia anterior, con un caudal informativo más voluminoso, siendo los discursos docentes auspiciadores de estas perspectivas, que en la realidad diaria no se concretan, más que sólo en el pensar y en la aplicación de ciertas metodologías ocasionales, excluyendo de los propósitos, aquello que verdaderamente permite cualificar al aprender como más significativo y valeroso. Pero quizás lo más interesante de todas estas conclusiones es la utilidad que proporcionan, ya que al acompañar la clase con instancias de ejercicios favorecedores del conocimiento del propio canal de aprendizaje, se podrá promocionar el autoconocimiento y la autonomía necesarias para brindar una formación integral y transformar al alumno en un estudiante estratega, capaz de resolver de manera independiente los obstáculos que el aprendizaje le depare.

\section{Agradecimientos}

A todos los estudiantes que participaron de esta investigación y a los colaboradores de cada asignatura que forman parte de este proyecto.

\section{Referencias}

Burón, J. (1997). Enseñar a aprender. Introducción a la metacognición. Bilbao: Ediciones Mensajero.

Camacho Segura, R. (2007). El proceso de enseñanza-aprendizaje: Manos arriba. México: ST.

Campayo, R.( 2004). Desarrolla una mente prodigiosa. Madrid: Ed Edaf S. A.

Creswell, J. (2008). Mixed Methods Research: State of the Art.Power Point Presentation.University of Michigan.

Recuperadodesitemaker.umich.edu/creswell.workshop/files/creswell lecture slides.ppt Dunn, R., Dunn, K. And Price, G. (1985). Manual: Learning Style Inventory. Lawrence, KS: Price Systems. 
Fascendini, P. Y. (2016) .Estrategias metacognitivas ¿cómo aprende el que aprende?. Alemania: Ed. Académica Española.

Fernandez Coto, R.(2012). Cerebrando el aprendizaje. Bs. As: Editorial Bonum.

Mora, F. (2017). Neuroeducación: solo se puede aprender aquello que se ama. Argentina: Alianza.

Tylbor, F. (2014).Estrategias para estudiar (EPE). Bs.As.: Ed Novedades Educativas. 